\title{
DIGOXINA E VERAPAMIL INDUZEM HIPERTROFIA EM CARDIOMIÓCITOS DE RATOS SEDENTÁRIOS E/OU TREINADOS
}

\author{
DIGOXIN AND VERAPAMIL PROMOTE CARDIOMYOCYTE HYPERTROPHY IN SEDENTARY \\ AND/ORTRAINED RATS
}

DIGOXINA Y VERAPAMILO INDUCEN HIPERTROFIA DE CARDIOMIOCITOS EN RATAS SEDENTARIAS Y/OENTRENADAS

\author{
Claodete Hasselstrom Neves \\ (Educadora Física) \\ Andreo Fernando Aguiar² \\ (Educador Físico) \\ Danilo Henrique Aguiar' (Biólogo) \\ André Soares Leopoldo 3 \\ (Educador físico) \\ Ana Paula Lima-Leopoldo ${ }^{3}$ \\ (Educadora física) \\ Fabrício Azevedo Voltarelli ${ }^{4}$ \\ (Educador físico) \\ Fabio José Lourenço \\ (Médico veterinário) \\ Mario Mateus Sugizaki \\ (Educador físico)
}

1. Instituto de Ciências da Saúde, Universidade Federal de Mato Grosso. Sinop, MT, Brasil.

2. Centro de Ciências Biológicas, Universidade do Norte do Paraná, Londrina, PR, Brasil.

3. Centro de Educação Física e Desportos, Universidade Federal de Espírito Santo, Vitória, ES, Brasil.

4. Departamento de Educação Física, Universidade Federal de Mato Grosso. Cuiabá, MT Brasil.

\section{Correspondência:}

Mário Mateus Sugizaki

Instituto de Ciências da Saúde Universidade Federal de Mato Grosso. Av. Alexandre Ferronato 1200, Sinop, Cuiabá, MT, Brasil. 78557-267

mario.ufmt@gmail.com

\begin{abstract}
RESUMO
Introdução: Cardiotônicos e bloqueadores de canais de cálcio são fármacos que alteram o Ca2+ intracelular e afetam o coração. Objetivo: Avaliar os efeitos da administração de verapamil e digoxina sobre a morfologia cardíaca de ratos submetidos ao treinamento intervalado (TAl). Métodos: Ratos Wistar machos divididos em seis grupos ( $\mathrm{N}=8$ ): Controle, Digoxina (30,0 $\mathrm{mg} . \mathrm{kg}-1 / \mathrm{dia})$, Verapamil (5,0 mg.kg-1/dia), Treinado, Treinado+digoxina e Treinado+verapamil. O TAl foi realizado em esteira rolante $(60 \mathrm{~min} / \mathrm{dia} / 60 \mathrm{dias})$ concomitantemente com a administração dos fármacos. Fragmentos do ventrículo esquerdo (VE) foram coletados para análise histológica. Resultados: A digoxina e o verapamil aumentaram a área total do $\mathrm{VE}(p<0,002)$, capilares/área $\mathrm{VE}(\mathrm{p}<0,01)$ e área de cardiomiócitos ( $p<2,8 \mathrm{e}-10)$, sendo que, nesta última variável, o verapamil promoveu efeito ainda maior que a digoxina. O TAl aumentou VE/PC ( $p<4$-05), o diâmetro interno do VE ( $p<2,7 e-6)$, a área de cardiomiócitos $(p<1,8$ e-6) e reduziu o [Lac] ( $p<2,6 e-5)$. Houve interação entre TAl e fármacos na área total $(p<9,8$ e-5), capilares $(p<0,04)$, células/área $(p<0,004)$ e área de cardiomiócitos $(p<2 e-16)$. Conclusão: A digoxina promoveu hipertrofia de cardiomiócitos e, quando associada ao TAl, potencializou a hipertrofia. O verapamil foi mais eficiente em aumentar a área de cardiomiócitos em comparação com a digoxina, porém somente de forma isolada.
\end{abstract}

Descritores: cardiotônicos; bloqueadores dos canais de cálcio; atividade motora; coração.

\section{ABSTRACT}

Introduction: Cardiotonics and calcium channel blockers are drugs that alter intracellular $\mathrm{Ca} 2+$ and can affect the heart. Objective: To evaluate the effects of administration of verapamil and digoxin on heart morphology of rats subjected

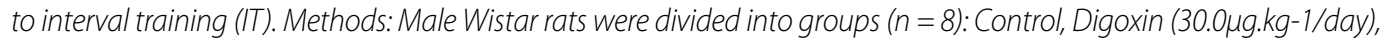
Verapamil (5.0 mg.kg-1/day), Trained, Trained+digoxin and Trained+verapamil. The IT was performed on a treadmill (60 min/day/60 days) concurrently with the drugs administration. Fragments of the left ventricle ( $L V$ ) were collected for histological analysis. Results: Digoxin and verapamil increased the total area of the LV ( $p<0.002)$, capillary/LV area $(p<0.01)$ and cardiomyocytes area $(p<2.8 \mathrm{e}-10)$, and in the latter variable, verapamil promoted even greater effect than digoxin. The IT increased LV/BW ( $p<4 e-05)$, the inner diameter of the LV ( $p<2.7 e-6)$, the area of cardiomyocytes $(p<1.8 e-6)$, and reduced the $[\mathrm{LaC}](p<2.6 e-5)$. There was interaction between IT and drugs in the total area $(p<9.8 e-5)$, capillaries $(p<0.04)$, cell/area $(p<0.004)$ and cardiomyocytes area $(p<2.0 e-16)$. Conclusions: Digoxin promoted cardiomyocyte hypertrophy and when associated with IT, potentiated the hypertrophy. Verapamil was more efficient in increasing the cardiomyocytes area compared with digoxin, but only when isolated.

Keywords: cardiotonic agents; calcium channel blockers; motor activity; heart.

\section{RESUMEN}

Introducción: Cardiotónicos y bloqueadores de los canales de calcio son fármacos que alteran el Ca2+ intracelulary afectan al corazón. Objetivo: Evaluar los efectos de la administración de verapamilo y digoxina sobre la morfología del corazón de ratas sometidas a entrenamiento a intervalos (EI). Métodos: Ratas Wistar macho, divididas en seis grupos ( $N=8)$ : Control, Digoxina (30,0 Mg.kg-1/dia), Verapamilo (5,0 mg.kg-1/día), Entrenado, Entrenado+digoxina y Entrenado+verapamilo. El entrenamiento a intervalos se realizó en una cinta de correr (60 min/día/60 días), con la administración concomitante de fármacos. Se recogieron fragmentos del ventrículo izquierdo (VI) para el análisis histológico. Resultados: La digoxina y el verapamilo aumentaron el área total del VI $(p<0,002)$, capilares/área VI $(p<0,01)$ y el área de los cardiomiocitos $(p<2,8 e-10)$ y, en esta última variable, el verapamilo promovió un efecto aún mayor que la digoxina. El entrenamiento a intervalos aumentó VI/PC ( $p<4 e-05)$, el diámetro interior del VI $(p<2,7 e-6)$, el área de los cardiomiocitos $(p<1,8 e-6)$ y redujo el [LaC] $(p<2,6 e-5)$. Hubo una interacción entre fármacos y el El en el área total $(p<9,8 e-5)$, capilares $(p<0,04)$, células/área $(p<0,004)$ y el área de los cardiomiocitos $(p<2 e-16)$. Conclusión: La digoxina promovió la hipertrofia de los cardiomiocitos y, cuando al asociarse con el El, potenció la hipertrofia. El verapamilo fue más eficiente en el aumento de la zona de los cardiomiocitos en comparación con la digoxina, pero sólo de forma aislada.

Descriptores: cardiotónicos; bloqueadores de los canales de calcio; actividad motora; corazón. 


\section{INTRODUÇÃO}

Os cardiotônicos e os bloqueadores de canais de cálcio (BCCs) são fármacos comumente utilizados em pacientes com cardiopatias. Os primeiros aumentam a força de contração cardíaca por elevarem os níveis citosólicos de cálcio $\left(\mathrm{Ca}^{2+}\right)^{1,2}$. O mecanismo de ação proposto mais aceito é que o cardiotônico se liga à bomba de sódio/potássio $\left(\mathrm{Na}^{+} / \mathrm{K}^{+}\right)$ da membrana do cardiomiócito elevando os níveis de $\mathrm{Na}^{+}$intracelular e, consequentemente, o trocador $\mathrm{Na}^{+} / \mathrm{Ca}^{2+}$ promove a troca de $\mathrm{Ca}^{2+}$ para dentro da célula e o $\mathrm{Na}^{+}$para fora. Os BCCs promovem efeitos inversos dos cardiotônicos. Ao se ligarem aos canais de $\mathrm{Ca}^{2+}$ voltagem dependente, eles bloqueiam o influxo de $\mathrm{Ca}^{2+}$ promovendo assim, redução tanto da contratilidade como da condutibilidade cardíaca. Desta forma, esses fármacos alteram a biodisponibilidade intracelular de $\mathrm{Ca}^{2+}$ no cardiomiócito determinando o grau de força de contração muscular. Entretanto, o $\mathrm{Ca}^{+2}$ participa de outros processos fisiológicos, incluindo a transcrição gênica e a proliferação celular ${ }^{3,4}$.

Não estão bem elucidados na literatura os efeitos desses fármacos sobre a morfologia cardíaca de corações normais ou hipertrofiados pelo treinamento físico (TF). Uma vez que o TF promove efeitos benéficos ao coração, como melhora do débito cardíaco no exercício, melhora no controle do sistema nervoso autônomo, hiperplasia e aumento no número de mitocôndrias, nossa hipótese é que a administração crônica de cardiotônico ou BCCs, associada ao treinamento físico intervalado de alta intensidade (TAl), promova alterações na estrutura das células cardíacas. O objetivo desse trabalho foi avaliar os efeitos da administração crônica de verapamil ou digoxina sobre a morfologia cardíaca de ratos submetidos ao TAI.

\section{METODOLOGIA}

\section{Animais e grupos experimentais}

Foram utilizados 48 ratos Wistar, machos, com 60 dias de idade. Os animais foram mantidos em gaiolas coletivas ( 4 animais/gaiola) em ambiente com temperatura controlada $\left(24 \pm 2^{\circ} \mathrm{C}\right)$ sob um ciclo claroescuro de 12h, alimentados com ração comercial Labina (Purina, Paulínia, SP, Brasil) e água ad libitum ${ }^{5}$. Este estudo foi aprovado pelo Comitê de Ética em Pesquisa Animal da Universidade Federal de Mato Grosso (CEPA - UFMT) (protocolo no: 23108.019254/11-0) e foi conduzido de acordo com as normas estabelecidas no "Guide for the Care and Use of Laboratory Animals" e Princípios Éticos na Experimentação Animal do Colégio Brasileiro de Experimentação Animal.

Os ratos foram aleatoriamente divididos em $(\mathrm{N}=8)$ : controle (C), treinado (T), digoxina (DIGO), verapamil (VERA), treinado mais digoxina (TDIGO) e treinado mais verapamil (TVERA). A dose de digoxina (Pharlab Indústria Farmacêutica, Lagoa da Prata-MG) foi de 30,0 $\mu \mathrm{gg} . \mathrm{kg}^{-1}$ e a dose de verapamil foi de $5,0 \mathrm{mg} . \mathrm{kg}^{-1}$, sendo que ambas foram administradas por gavagem, uma vez ao dia por 75 dias. A concentração de digoxina utilizada nesse trabalho baseou-se em Wang et al. ${ }^{1}$ e Manunta et al. ${ }^{6}$ Os grupos C e T foram submetidos ao mesmo procedimento da gavagem, porém, receberam apenas solução fisiológica para garantir as mesmas condições a todos os grupos.

\section{Treinamento físico intervalado de alta intensidade (TAI)}

Os grupos T, TDIGO e TVERA foram submetidos a um protocolo de adaptação à esteira, com duração de uma semana (velocidade de $10 \mathrm{~m} / \mathrm{mim}, 15 \mathrm{~min}$ por dia). Posteriormente, os grupos T, TVERA e TDIGO foram submetidos a um teste de esforço máximo (TEM) realizado em esteira rolante, o qual foi repetido após 30 e 60 dias do início do treinamento. O TEM iniciou com velocidade de $10 \mathrm{~m} / \mathrm{min}$ e aumentos progressivos de 2,0 m/min cada dois minutos até a exaustão. 0 critério de exaustão adotado na avaliação no ergômetro foi a não manutenção da corrida na velocidade proposta por cinco segundos.
O protocolo de treinamento físico por nós utilizado foi adaptado de Kemi et al.?, uma vez que tem sido proposto como uma estratégia adequada para promover respostas benéficas sobre a função e hipertrofia cardíaca8. Após o pré-teste, os grupos T, TVERA e TDIGO foram submetidos TAl em esteira rolante (8 min em velocidade correspondente à $80 \%$ da Vmáx e dois min em velocidade correspondente à $20 \%$ da $V_{\text {máx }}$ ) durante 60 min. O tempo total de treinamento foi de oito semanas ininterruptas.

\section{Determinação da concentração de lactato sanguíneo}

Para avaliar a eficácia do protocolo de TAI, foi determinada a concentração de lactato sanguíneo imediatamente após o teste de esforço máximo utilizando lactímetro portátil (Roche, Alemanha). Para cada teste foi coletada uma gota de sangue da extremidade da cauda do animal.

\section{Dados anatômicos e perfil nutricional}

Ao término do período experimental, os animais foram eutanasiados, o coração foi imediatamente retirado e os ventrículos direito (VD) e esquerdo (VE) foram separados dos átrios; os tecidos adiposos foram excisados e totalmente retirados. Todas as amostras supracitadas foram pesadas em balança analítica (Marca Shimadzu, Alemanha).

Fragmentos do VE foram colocados em solução de cloreto de potássio (KCl) para que o ciclo cardíaco fosse interrompido em diástole; além disso, foram medidas a espessura da parede do VE (EPVE) e o diâmetro interno do VE (DIVE) com o auxílio de um paquímetro de aço inox (Jomarca-China) e, em seguida, o VE foi colocado em solução de fixador para posterior análise histológica.

\section{Análise histológica}

Após ser fixado em paraformaldeído 10\% tamponado, o VE foi submetido ao processamento para a análise histológica, com cortes de três $\mu \mathrm{m}$ e coloração PAS (ácido periódico+reativo de Schiff) com a solução de metanilyellow). Todas as análises foram realizadas por um único avaliador cego para o grupo de imagens.

Para a análise, foi utilizado um sistema computadorizado acoplado ao microscópio ótico com aumento de 20x. Foram selecionados quatro campos da região endocárdica-miocárdica (aproximadamente 200 células) para cada animal. Foi medido em $\mu \mathrm{m}^{2}$ a área total do campo, o diâmetro da área celular de cada cardiomiócito e foram contados os números de cardiomiócitos e de capilares.

\section{Análise estatística}

As variáveis foram expressas por meio de medidas descritivas de posição e variabilidade. A comparação dos grupos experimentais foi realizada pela técnica de Kruskal Wallis para o esquema de dois fatores independentes (droga e treinamento), utilizando-se o software R. O índice de significância considerado para todas as variáveis foi de $5 \%$.

\section{RESULTADOS}

A Tabela 1 apresenta as características gerais dos animais dos grupos experimentais. Não houve diferença significante entre os grupos para as variáveis, peso corporal e consumo de ração, indicando que a administração crônica de digoxina ou de verapamil associadas ou não ao TAl, não afetaram o crescimento somático dos animais.

De acordo com os dados da Tabela 2, as drogas utilizadas afetaram a relação VE/PCF $(p<0,04)$. O TAI apresentou aumento no VE/ PCF $\left(p<4,0 e^{-5}\right)$ e DIVE $\left(p<2,7 e^{-6}\right)$ e diminuição na GOR/PCR $\left(p<2,9 e^{-5}\right)$. Houve interação entre as drogas e TAl no VE/PCF $(p<0,0002)$, GOR/PCF $(p<0,0004)$ e DIVE $\left(p<9,3 e^{-5}\right)$

O teste de esforço máximo foi utilizado para comprovar a eficácia do TAI. A Figura 1A e 1B demonstra que todos os grupos treinados 
aumentaram o tempo total e a distância total percorrida do momento pré para o pós 60 dias de TAI. No entanto, este aumento foi similar entre os grupos em todos os momentos, sugerindo que as drogas utilizadas não afetaram o desempenho físico.

A Figura 2 representa a concentração plasmática de lactato imediatamente após o TEM após 60 dias de TAl. Observou-se efeito do treinamento $\left(p<2,6 e^{-5}\right)$ sendo que o grupo T foi menor que o grupo $C, 0$ grupo TDIGO foi menor que o grupo DIGO e o grupo TVERA foi menor que o grupo VERA.

A Tabela 3 apresenta os dados histológicos do ventrículo esquerdo dos grupos experimentais. Observou-se efeito das drogas na área total ( $p<0,002)$, no número de capilares/área $(p<0,016)$ e na área de cardiomiócito $\left(p<2,83 e^{-10}\right)$ sendo que nesta última variável, o verapamil teve efeito maior que a digoxina. OTAl aumentou a área de cardiomiócito $\left(p<1,85 e^{-6}\right)$. Houve interação entre as drogas e o TAI na área total do VE $\left(0,79 e^{-5}\right)$, no número de capilares $(p<0,04)$, no número de capilares/área $(p<0,006)$, no número de células/área $(p<0,004)$ e na área de cardiomiócito $(p<0,00)$.

As imagens histológicas de tecido do ventrículo esquerdo (Figura 3) demostram que os cardiomiócitos estão preservados, com ausência de lesão, dano celular, fibrose, núcleos centralizados, células endoteliais e capilares aparentemente normais.

\section{DISCUSSÃO}

O objetivo desse estudo foi avaliar os efeitos da administração crônica de cardiotônico ou BCCs sobre a morfologia de cardiomiócitos de ratos submetidos ao TAl. O TAl melhorou a aptidão física dos animais pertencentes a todos os grupos treinados, sendo isto confirmado pelos resultados obtidos no TEM; sugerimos que tal melhora possa estar relacionada à otimização tanto da capacidade oxidativa muscular como das capacidades cardiocirculatória e neuroendócrina advindas do treinamento físico. Além disso, os menores níveis de lactato sanguíneo apresentados pelo grupo T imediatamente após o TEM sugerem, ao menos em parte, ocorrência de adaptação metabólica ao treinamento físico (i.e., menor produção e/ou aumento na taxa de remoção do lactato produzido), corroborando, desta forma, o estudo de Menezes et al. ${ }^{9}$, os quais também verificaram essa redução. Os mecanismos responsáveis por esse fenômeno estão relacionados à maior oxidação de ácidos graxos bem como à redução na magnitude de funcionamento da glicólise anaeróbia e, ainda, de forma importante, à conversão do lactato em glicogênio (neoglicogênese), principalmente no tecido hepático ${ }^{10}$.

Clinicamente, a dosagem de digoxina usualmente empregada é de 0,25 mg para pacientes com função renal normal. Isso equivale à concentração de 3,57 $\mu \mathrm{g} \cdot \mathrm{kg}^{-1}$ em um paciente de 70 kg, correspondendo

Tabela 1. Características gerais dos animais.

\begin{tabular}{c|c|c|c|c|c|c}
\hline & $\begin{array}{c}\mathbf{C} \\
\mathbf{N = 7}\end{array}$ & $\begin{array}{c}\text { DIGO } \\
\mathbf{N = 6}\end{array}$ & $\begin{array}{c}\text { VERA } \\
\mathbf{N = 7}\end{array}$ & $\begin{array}{c}\mathbf{T} \\
\mathbf{N = 8}\end{array}$ & $\begin{array}{c}\text { TDIGO } \\
\mathbf{N = 7}\end{array}$ & $\begin{array}{c}\text { TVERA } \\
\mathbf{N = 8}\end{array}$ \\
\hline $\mathrm{PCl}(\mathrm{g})$ & $245 \pm 22$ & $229 \pm 28$ & $217 \pm 39$ & $242 \pm 18$ & $215 \pm 39$ & $206 \pm 32$ \\
\hline $\mathrm{PCF}(\mathrm{g})$ & $369 \pm 23$ & $374 \pm 23$ & $370 \pm 33$ & $373 \pm 22$ & $351 \pm 42$ & $320 \pm 35$ \\
\hline
\end{tabular}

\begin{tabular}{l|l|l|l|l|l|l|l|}
\hline Consumo ração $(\mathrm{g})$ & $23,3 \pm 0,2$ & $23,8 \pm 1,6$ & $24,1 \pm 0,7$ & $25,8 \pm 0,2$ & $24,5 \pm 1,5$ & $23,2 \pm 1,1$ \\
\hline
\end{tabular} Valores expressos em média \pm desvio padrão; C: controle; T: treinado; DIGO: digoxina; TDIGO: treinado+digoxina; VERA: verapamil;TVERA: treinado+ verapamil; PCI: peso corporal inicial; PCF: peso corporal final; Kruskal Wallis, $\mathrm{p}<0,05$ a 10 vezes menos a dosagem que empregada por nós $\left(30 \mu \mathrm{g} \cdot \mathrm{kg}^{-1}\right)$. A dosagem de verapamil utilizada nesse estudo foi de $5,0 \mathrm{mg} \cdot \mathrm{kg}^{-1}$, sendo esta a mesma empregada clinicamente para pacientes hipertensos e corrobora com outros estudos experimentais ${ }^{11,12}$. Para verificar a possível toxicidade das drogas utilizadas, nós avaliamos os parâmetros séricos de creatinina, TGO, TGP e proteínas totais (dados não apresentados). Uma vez que não houve alteração nesses parâmetros, consideramos que as drogas, isoladamente ou associada ao TAl, não causaram prejuízo à função renal ou hepática dos animais.

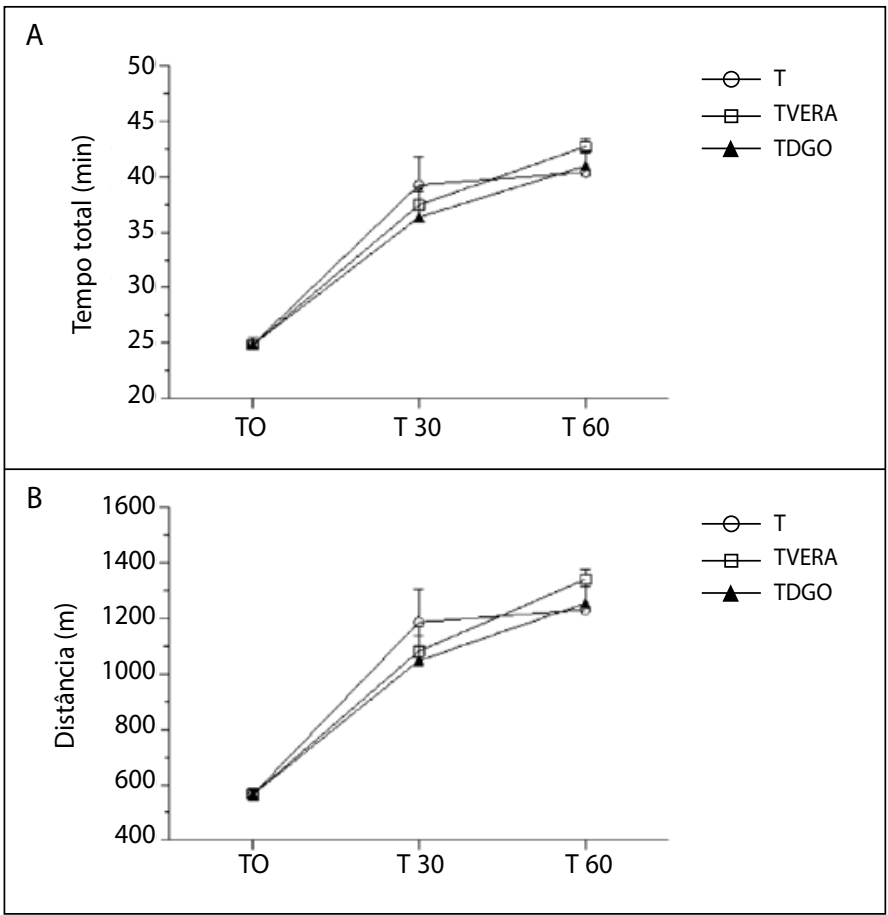

Figura 1. Teste de esforço em ratos treinados (T); Treinado+digoxina (TDIGO) e Treinado+verapamil (TVERA), antes do treinamento intervalado (T0) após 30 dias (T30) e após 60 dias (T60). A: tempo total de teste (min). B: distância total percorrida (m). Dados expressos em média \pm erro padrão da média.

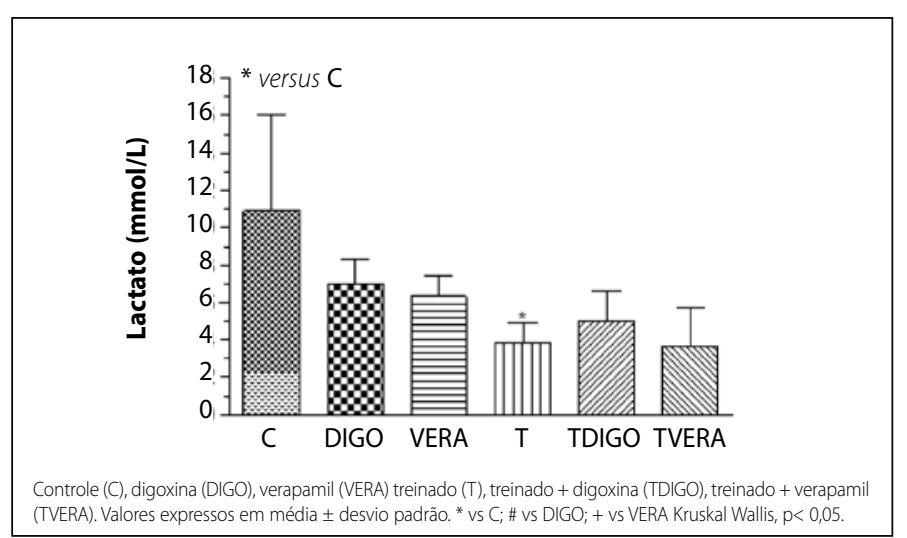

Figura 2. Concentração plasmática de lactato após o teste de esforço máximo no momento pós-treinamento. ( $\mathrm{N}=8$ por grupo).

Tabela 2. Relações entre gordura corporal, músculo cardíaco com o peso corporal final, espessura da parede e diâmetro interno do ventrículo esquerdo.

\begin{tabular}{c|c|c|c|c|c|c}
\hline & $\begin{array}{c}\mathbf{C} \\
\mathbf{N = 7}\end{array}$ & $\begin{array}{c}\text { DIGO } \\
\mathbf{N = 6}\end{array}$ & $\begin{array}{c}\text { VERA } \\
\mathbf{N = 7}\end{array}$ & $\begin{array}{c}\text { T } \\
\mathbf{N = 8}\end{array}$ & $\begin{array}{c}\text { TDIGO } \\
\mathbf{N = 7}\end{array}$ & $\begin{array}{c}\text { TVERA } \\
\mathbf{N = 8}\end{array}$ \\
\hline Gor/PCF & $39,4 \pm 3,9$ & $42,4 \pm 6,5$ & $41,0 \pm 5,8$ & $35,4 \pm 6,4$ & $31,0 \pm 3,6 \# \neq$ & $29,2 \pm 3,6+\ddagger$ \\
\hline VE/PCF & $1,92 \pm 0,05$ & $2,10 \pm 0,21^{*}$ & $2,01 \pm 0,11$ & $2,12 \pm 0,06^{*}$ & $2,29 \pm 0,19 \#$ & $2,37 \pm 0,19+\ddagger$ \\
\hline VD/PCF & $0,565 \pm 0,067$ & $0,602 \pm 0,073$ & $0,603 \pm 0,06$ & $0,634 \pm 0,06$ & $0,660 \pm 0,08$ & $0,684 \pm 0,099^{*}$ \\
\hline EPVE & $0,291 \pm 0,033$ & $0,308 \pm 0,016$ & $0,282 \pm 0,050$ & $0,341 \pm 0,052$ & $0,297 \pm 0,071$ & $0,323 \pm 0,040$ \\
\hline DIVE & $0,244 \pm 0,065$ & $0,301 \pm 0,046$ & $0,294 \pm 0,037$ & $0,377 \pm 0,080^{*}$ & $0,461 \pm 0,121 \# \neq$ & $0,383 \pm 0,075+$ \\
\hline
\end{tabular}

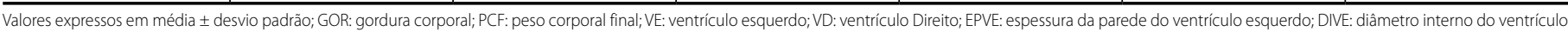
esquerdo. * vs C; \# vs DIGO; + vs VERA; \# vs T; Kruskal Wallis, $p<0,05$ 
Tabela 3. Dados histológicos ventrículo esquerdo.

\begin{tabular}{|c|c|c|c|c|c|c|}
\hline & C & DIGO & VERA & $\mathrm{T}$ & TDIGO & TVERA \\
\hline Área total $(\mu 2)$ & $32645 \pm 7797$ & $37527 \pm 9121^{*}$ & $45079 \pm 6937^{*} \#$ & $41684 \pm 7013^{*}$ & $38967 \pm 6190$ & $41738 \pm 6164$ \\
\hline № de capilares & $35,1 \pm 12,0$ & $35,9 \pm 12,1$ & $39,1 \pm 14,0$ & $40,9 \pm 13,7$ & $28,7 \pm 12,6 \# \neq$ & $37,2 \pm 10,9$ \\
\hline № de células & $51,9 \pm 10,7$ & $51,4 \pm 14,6$ & $58,0 \pm 13,9$ & $55,1 \pm 12,6$ & $53,8 \pm 10,5$ & $56,6 \pm 12,4$ \\
\hline Área cardiomiócito $(\mu 2)$ & $563 \pm 205$ & $615 \pm 243^{*}$ & $692 \pm 248^{*} \#$ & $666 \pm 254^{*}$ & $682 \pm 278 \#+$ & $648 \pm 261 \#++$ \\
\hline Células/área $\left(10^{-4}\right)$ & $16,1 \pm 2,3$ & $13,8 \pm 2,8^{*}$ & $12,8 \pm 2,0^{*}$ & $13,2 \pm 2,2^{*}$ & $14,1 \pm 3,3^{*}$ & $13,7 \pm 2,9^{*}$ \\
\hline Capilares/área $\left(10^{-4}\right)$ & $10,7 \pm 2,5$ & $9,6 \pm 2,5$ & $8,3 \pm 3,3^{*}$ & $9,9 \pm 3,0$ & $7,4 \pm 3,2^{*} \# \neq$ & $9,0 \pm 2,8+$ \\
\hline
\end{tabular}
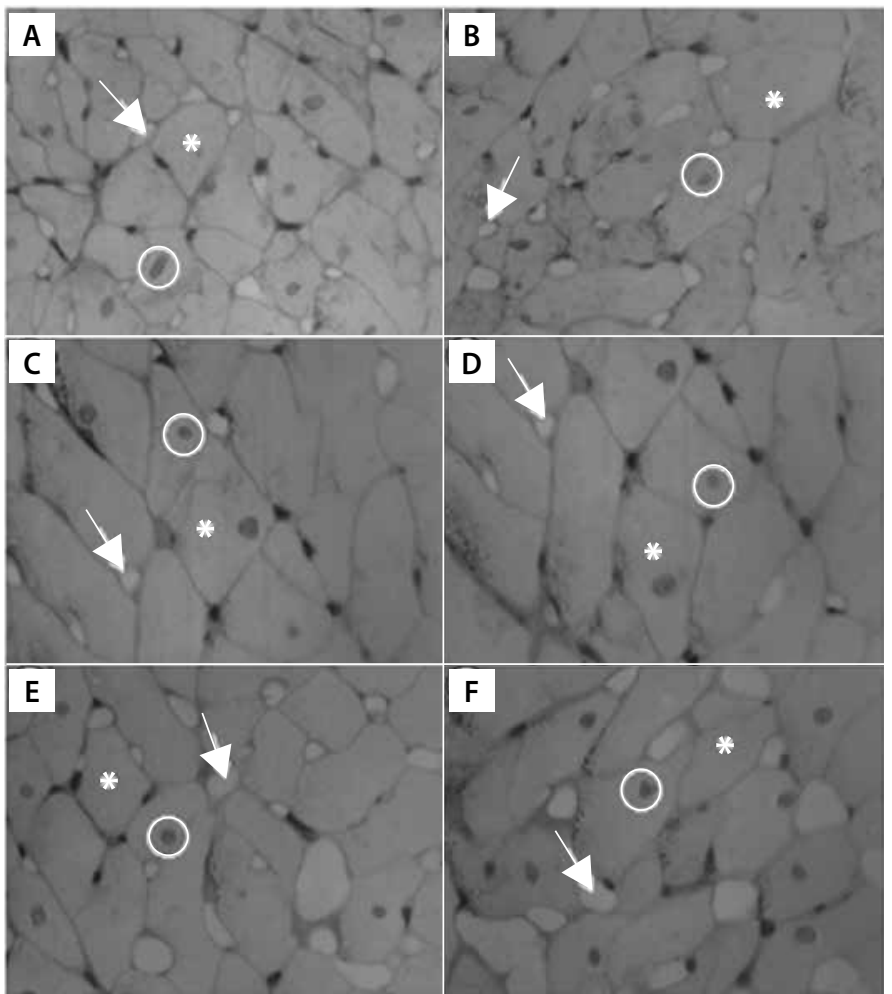

Figura 3. Corte transversal da região endocárdica-miocárdica do ventrículo esquerdo. A) Controle; B) Treinado; C) Digoxina; D) Treinado+digoxina; E)Verapamil; F) Treinado+verapamil. Observam-se os cardiomiócitos (asterisco), capilares (setas) e núcleo (círculo). Coloração PAS+ metanilyellow. Aum. 40x.

Nossos achados relacionados à hipertrofia cardíaca mostraram que o treinamento empregado promoveu aumento no diâmetro do VE e da área do cardiomiócito, corroborando com outros importantes estudos $^{13}$. Sabe-se que o tipo, a frequência, a intensidade e a duração do TF promovem diferentes adaptações na função cardíaca e dimensões ventriculares ${ }^{14}$. As adaptações morfológicas cardíacas correspondem à natureza dos estímulos hemodinâmicos (sobrecarga de pressão ou sobrecarga de volume) impostos ao miocárdio durante sessões repetidas de exercício e a modificação da relação entre a espessura da parede e o diâmetro do ventrículo hipertrófico deve obedecer a lei de Laplace ${ }^{15}$. O protocolo de exercício utilizado em nosso estudo foi o modelo intervalado de alta e baixa intensidade e longa duração, este caracterizado por sobrecarga de volume induzindo hipertrofia do tipo excêntrica (DIVE aumentado) ${ }^{13}$.

Nossos achados de área de cardiomiócito indicam que administração crônica de digoxina induziu hipertrofia cardíaca e, quando associada ao treinamento físico, os resultados foram ainda mais proeminentes. A digoxina é utilizada na insuficiência cardíaca por aumentar a disponibilidade intracelular de $\mathrm{Ca}^{2+}$ no cardiomiócito aumentando o grau de força de contração muscular. Além do efeito inotrópico positivo, os cardiotônicos possuem efeitos neuro-endócrinos, neuro-hormonais e eletrofiológicos, os quais podem causar interferencia no processo hipertrófico ${ }^{16}$. Os cardiotônicos não alteram o débito cardíaco em sujeitos normais, porém, em pacientes com reduzida função sistólica, estes fármacos melhoram a fração de ejeção do ventrículo esquerdo e aumentam o débito cardíaco em repouso e durante o exercício ${ }^{17}$. Nossos achados divergem com os obtidos no trabalho de Aldinger ${ }^{18}$, o qual não verificou hipertrofia cardíaca em pacientes tratados com digoxina e submetidos ao treinamento físico intermitente.

Os mecanismos moleculares envolvidos na hipertrofia de cardiomiócito induzida pela digoxina podem estar relacionados ao aumento da disponibilidade de cálcio intracelular. Recentes evidências sugerem que a resposta hipertrófica ao cálcio está associada à ativação da proteína quinase C (PKC), cálcio-calmodulina quinase II (CAMKII) e calcineurina fosfatase (CaN) ativada pelo complexo cálcio-calmodulina ${ }^{2,4}$. A ativação da PKC e CAMKII fosforila fatores transcricionais nucleares e a CaN causa defosforilação e ativação das células T ativadas por fatores nucleares (NFAT), as quais estão localizadas no citoplasma quando é fosforilada em condições basais. Após a defosforilzação, NFAT transloca-se para a região promotora nuclear promovendo ativação de genes musculo-específicos e promovendo, consequentemente, hipertrofia cardíaca ${ }^{2,19}$. Tem sido bem documentado que o treinamento físico induz hipertrofia ventricular e aumento do transiente de $\mathrm{Ca}^{2+20}$. Dessa forma, nossos achados de hipertrofia de cardiomiócito induzidos pela digoxina podem estar relacionados às alterações de disponibilidade de cálcio intracelular. Novos estudos necessitam ser realizados para avaliar o transiente de cálcio e a ativação dessas vias de sinalização intracelular.

Interessantemente, a administração crônica de verapamil promoveu aumento da área do cardiomiócito. Entretanto, quando associado ao treinamento físico, esse efeito não foi observado. Os BCCs promovem efeito inotrópico negativo, uma vez que diminuem a força de contração cardíaca por reduzir a disponibilidade de cálcio intracelular. Seu efeito mais importante como anti-hipertensivo reside na sua ação vasodilatadora, resultando em diminuição da pressão arterial sistêmica. Contrariamente aos nossos achados, dados da literatura sugerem que os BCCs podem atenuar o desenvolvimento da hipertrofia cardíaca em ratos espontaneamente hipertensos ${ }^{11,12}$. Essa divergência de resultados pode ser atribuída às condições clínicas dos ratos, uma vez que utilizamos animais saudáveis e, de acordo com alguns autores, os níveis de verapamil nos tecidos do coração diferem drasticamente entre ratos normotensos e hipertensos ${ }^{21,22}$. Não encontramos dados na literatura que suportam a ideia de aumento de área de cardiomiócito induzida por BCCs em condições de normotensão. Estudos in vitro e in vivo demonstraram que os BCCs induzem hiperglicemia por inibir a liberação de insulina nas células beta pancreática23,24. Em doses tóxicas, o verapamil pode promover efeito diabetogênico ${ }^{25}$. Além disso, tem sido demonstrada relação entre miocárdio hipertrófico e resistência à insulina, independentemente da presença de diabetes mellitus ${ }^{26}$.

As vias de sinalizacao referentes à resistencia insulínica no miocárdio são complexas e multifatoriais, pois estão envolvidas em anormalidades na homeostasia do calcio intracelular (canal L, fosfolamban, SERCA2A, trocador $\mathrm{Na}^{+} / \mathrm{Ca}^{+2}$ ) e sinalização da insulina (IRS-PI3K-Akt). Por outro lado, tem sido demonstrado que o treinamento físico reduz a resistencia insulínica ${ }^{27}$. Não apresentamos os dados de glicemia, 
entretanto verificamos aumento significativo $(p<0,0007)$ dos níveis séricos de glicose em ratos que receberam verapamil (95 \pm 8 ) em relação aos ratos tratados com verapamil e submetidos ao treinamento físico (76 10 ). A hiperglicemia e a resistência insulínica poderiam explicar os resultados da maior hipertrofia de cardiomiótico no grupo VERA em relação ao grupo TVERA. Novos estudos são necessários para a comprovação dessa hipótese por meio da dosagem de insulina sérica, da via de sinalização intracelular da insulina envovida na hipertrofia cardíaca em condições de hiperglicemia induzida pela administração crônica de verapamil.

Em relação à vascularização, nossos achados não verificaram alterações sobre a morfologia de vasos sanguíneos induzidos pelo treinamento ou administração de digoxina ou verapamil. Trabalhos têm mostrado que diversas situações como idade, condições clínicas e o próprio treinamento físico induzem angiogênese ${ }^{28}$. Roque et al. ${ }^{29}$ verificaram aumento do número de capilares de músculos papilares do coração de ratos submetidos ao treinamento de nata- ção por 10 semanas. Leosco et al..$^{30}$ verificaram que o treinamento em esteira por 10 semanas induziu angiogênese coronária em ratos com insuficiência cardíaca. Essa divergência de resultados pode estar relacionada à condição clínica do animal, ao modelo de treinamento empregado, à intensidade do exercício e a região cardíaca a qual foi submetida à análise.

\section{CONCLUSÃO}

Nossos achados sugerem que a administração crônica de digoxina ou de verapamil promoveu hipertrofia de cardiomiócitos de ratos sedentários e/ou treinados. Novos estudos devem ser realizados para a elucidação de possíveis mecanismos relacionados a esses importantes e significativos efeitos.

Todos os autores declararam não haver qualquer potencial conflito de interesses referente a este artigo.

CONTRIBUIÇÕES DOS AUTORES: Cada autor contribuiu individualmente e significativamente para o desenvolvimento do manuscrito. CHN (0000-0003-1173-7545)* contribuiu especificamente na concepção e desenho da pesquisa, obtenção, análise e interpretação dos dados e redação do artigo. AFA (0000-0003-3377-2444)* participou do estudo e contribuiu especificamente na análise e interpretação dos dados e redação do artigo. DHA (0000-0001-9791-9774)* participou do estudo e contribuiu especificamente na obtenção dos dados e análises histológicas. ASL (0000-0002-3131-2467)* e APLL (0000-0002-4049-4418)* participaram do estudo e contribuíram especificamente na revisão crítica do artigo. FAV (0000-0002-8077-8941)* participou do estudo e contribuiu especificamente na revisão e aprovação da versão final do artigo. FJL (0000-0003-2559-3028)* participou do estudo e contribuiu especificamente na análise estatística, revisão e aprovação da versão final do artigo. MMS (00000002-4513-0883)* contribuiu especificamente na análise e interpretação dos dados, análise estatística, revisão do artigo, concepção intelectual do artigo e elaboração do projeto de pesquisa. ${ }^{*}$ ORCID (Open Researcher and Contributor ID).

\section{REFERÊNCIAS}

1. Wang H, Yuan W, Lu Z. Effects of ouabain and digoxin on gene expression of sodium pump alpha-subunit isoforms in rat myocardium. Chin Med J (Engl). 2001; 114(10):1055-9.

2. Katz A, Lifshitz Y, Bab-Dinitz E, Kapri-Pardes E, Goldshleger R, Tal DM, et al. Selectivity of digitalis glycosides for isoforms of human $\mathrm{Na}^{+}$, K+-ATPase. J Biol Chem. 2010;285(25):19582-92.

3. Berridge MJ. Inositol trisphosphate and calcium signalling. Nature. 1993; 361(6410):315-25.

4. Clapham DE. Calcium signaling. Cell. 2007;131(6):1047-58.

5. Benevenga NJ. Calvert C, Eckhert CD, Fahey GC, Greger JL. Keen CL, et al. Nutrient requirements of the laboratory rat. In: Nutrient requirements of laboratory animals. 4nd ed. New York: National Academy Press. 1995. [acesso em 2014 mai 24]; Disponível em http://www.nap.edu/read/4758/chapter/4.

6. Manunta P, Hamilton J, Rogowski AC, Hamilton BP, Hamlyn JM. Chronic hypertension induced by oubain but not digoxin in the rat: Antihypertensive effect of digoxin and digitoxin. Hypertens Res. 2000;23(suppl):S77-85.

7. Kemi OJ, Ellingsen O, Ceci M, Grimaldi S, Smith GL, Condorelli G, et al. Aerobic interval training enhances cardiomyocyte contractility and $\mathrm{Ca}^{2+}$ cycling by phosphorylation of CaMKII and Thr-17 of phospholamban. J Mol Cell Cardiol. 2007; 43(3):354-61.

8. Kemi OJ, Ceci M, Wisloff U, Grimaldi S, Gallo P, Smith GL, et al. Activation or inactivation of cardiac Akt/ mTOR signaling diverges physiological from pathological hypertrophy. J Cell Physiol. 2008;214(2):316-21.

9. Menezes HS, Coracini JCD, Kepler KC, Frantz E, Abegg MP, Correa CA, et al. Ácido lático como indicativo de aptidão física em ratos. Rev Bras Med Esporte. 2010; 16(3):210-14

10. Brooks GA. Intra-and extra-cellular lactate shuttles. Med Sci Sports Exerc. 2000; 32(4):790-9

11. Min JY, Sandmann S, Meissner A, Unger T, Simon R. Differential effects of mibefradil, verapamil, and amlodipine on myocardial function and intracellular $\mathrm{Ca}^{2+}$ handling in rats with chronic myocardial infarction. J Pharmacol Exp Ther.1999; 291(3):1038-44.

12. Bombing MT, Luna Filho B, Costa EA, Leite DA, Póvoa R, Murad N, et al. Effect of verapamil on left ventricular hypertrophy induced by isoproterenol. Arq Bras Cardiol.1996;67(2):81-5.

13. Hashimoto NY, Fernandes T, Soci UPR, Oliveira EM. Determinantes moleculares da hipertrofia cardíaca induzida por diferentes volumes de treinamento aeróbio. Rev Bras Cardiol. 2011;24(3):153-62.

14. Weiner RB, Baggish AL. Exercise-induced cardiac remodeling. Prog Cardiovasc Dis. 2012;54(5):380-6.

15. Shapiro LM. The morphologic consequences of systemic training. Cardiol Clin. 1997;15(3):373-9.
16. Rahimtoola SH. Digitalis therapy for patients in clinical heart failure. Circulation. 2004;109(24):2942-6. 17. Gheorghiade M, Ferguson D. Digoxin. A neurohormonal modulator in heart failure? Circulation.1991;84(5):2181-6.

18. Aldinger EE. Effects of digitoxin on the development of cardiac hypertrophy in the rat subjected to chronic exercise. Am J Cardiol.1970;25(3):339-43.

19. Anderson ME. Calmodulin kinase signaling in heart: an intriguing candidate target for therapy of myocardial dysfunction and arrhythmias. Pharmacol Ther. 2005;106(1): 39-55.

20. Primola-Gomes TN, Campos LA, Lauton-Santos S, Balthazar CH, Guatimosim S, Capettini LS, et al. Exercise capacity is related to calcium transients in ventricular cardiomyocytes. J Appl Physiol. 2009; 107(2):593-8.

21. Zwadlo C, Borlak J. Impaired tissue clearance of verapamil in rat cardiac hypertrophy results in transcriptional repression of ion channels. Xenobiotica. 2010; 40 (4):291-9.

22. Michiels CF, Van Hove CE, Martinet W, De Meyer GR, Fransen P. L-type Ca ${ }^{2+}$ channel blockers inhibit the window contraction of mouse aorta segments with high affinity. Eur J Pharmacol. 2014;738:170-8

23. De Marinis $L$, Barbarino A. Calcium antagonists and hormone release. I. Effects of verapamil on insulin release in normal subjects and patients with islet-cell tumor. Metabolism.1980;29(7):599-604

24. Röjdmark S, Andersson DE. Influence of verapamil on human glucose tolerance. Am J Cardiol. 1986;57(7):39D-43D.

25. Kline JA, Raymond RM, Schroeder JD, Watts JA. The diabetogenic effects of acute verapamil poisoning Toxicol Appl Pharmacol. 1997;145(2):357-62.

26. Lebeche D, Davidoff AJ, Hajijar RJ. Interplay between impaired calcium regulation and insulin sign aling abnormalities in diabetic cardiomyopathy. Nat Clin pract Cardiovasc Med. 2008;5(11)715-24.

27. Borghouts LB, Keizer HA. Exercise and insulin sensitivity: a review. Int J Sports Med. 2000;21(1):1-12.

28. Prior BM, Yang HT, Terjung RL. What makes vessels grow with exercise training? J Appl Physiol. 2004;97(3):1119-28

29. Roque FR, Soci UP, De Angelis K, Coelho MA, Furstenau CR, Vassalo DV, et al. Moderate exercise training promotes adaptations in coronary blood flow and adenosine production in normotensive rats. Clinics. 2011;66(12):2105-11.

30. Leosco D, Rengo G, laccarino G, Golino L. Marchese M, Fortunato F, et al. Exercise promotes angiogenesis and improves beta-adrenergic receptor signalling in the post-ischaemic failing rat heart. Cardiovasc Res. 2008;78(2):385-94. 